\title{
High Impedance Fault Identification in TCSC-Compensated Power Transmission Lines
}

\author{
Mohammed Hussien Hassan Musa ${ }^{1,2, *}$, Abu Sabah I. A. Ahmed ${ }^{3}$ \\ 1 Electrical Engineering Department, Almughtaribeen University, Khartoum, Sudan \\ 2 Sudanese Thermal Power Generating Company, Khartoum, Sudan \\ 3 School of Electrical Engineering, Karari University, Sudan \\ * Corresponding author: Mohammed Hussien (seadamhd29@yahoo.com).
}

Article history: Received 23 October 2020, Received in revised form 06 December 2020, Accepted 23 December 2020

\begin{abstract}
The conventional distance relaying scheme performs poorly under the presence of Thyristors-controlled series capacitors (TCSC), and during the high impedance fault (HIF). TCSC provide a variable impedance during an internal/external fault and HIF is undetectable by the conventional overcurrent relaying due to low-current amplitude with non-linear behavior. Therefore, this paper presents a new Method for identifying HIF in TCSC-compensated transmission line. It is use the square error ration of the current signals during the fault period and current signals during the safe operating period for extracting the fault. The cumulative sum is being used for enlarging the fault features and then output index is obtained in order to perform the high impedance fault. The proposed method has been tested under different fault circumstances such as multiple fault locations, and multiple fault inception time. Moreover, fault happened nearby the terminal, power flow change, and faults in the presence of noise are also being considered. The Simulations are performed under different fault conditions using PSCAD/EMTDC and then the data is transferred to MATLAB environment to perform the fault detection process. The test results have shown that the proposed method is good in term of time response, and then it is more appropriate for high impedance fault in TCSC-compensated power line.
\end{abstract}

Keywords: Cumulative sum, Square error ratio, time response, high impedance fault, TCSC-compensated lines.

\section{INTRODUCTION}

The presence of TCSC devices in the fault path in power transmission line changes the system parameters, such as line impedance and load current. Because TCSC can provide a variable capacitive or inductive impedance. Therefore, the conventional distance schemes are adversely influenced by the presence of TCSC in the fault path $[1,2]$. The current resulted due to HIF may not sufficient to be detected by the over current protection [3]. Additionally, the random behavior, non-linearity and dependency towards network topology have added extra difficulties in HIF fault detection. Therefore, having a high impedance fault in TCSC compensated power transmission network need a precise and accurate scheme to satisfactory handling the faults in such dynamic situations.

Several schemes for fault identification in TCSC-compensated lines have been proposed; schemes in [4, 5] provided a technique for performing the fault detection based on the post-fault superimposed quantity. However, it is less reliable during High resistance fault. Additionally, [5] required a very high sampling rate to extract the transients. Time-frequency analysis based on discrete wavelet transform (DWT) of the measured three-phase current has 
been used for performing fault detection and classification in TCSC compensated line in [6]. The scheme in [7] discuss the fault analysis in TCSC compensated line with the help of discrete Wavelet Transform (DWT) and implementation of Chebyshev Neural Network (ChNN). A data-mining model for fault-zone identification is discussed in [8], Decision tree was used for fault zone identification and fault classification in [9]. However, schemes [7-10] utilizing specified sets of data to train their decision making algorithm. A travelling wave and Fast Discrete S-transform (FDST) have been used for detecting fault in TCSC compensated lines. However, the proposed method needs to have data acquisition system with a sampling rate of $200 \mathrm{kHz}$ [11]. The most susceptible schemes for high resistance faults are the pilot protection schemes as in [12]. These schemes used the synchronized samples of two terminals to assess the fault cases in power transmission lines [13]. However, the economic feasibility and complexity of these technology must be considered in designing and selecting such schemes. Usually back up earth-fault function is incorporated with distance protection to improve possible deficiencies of distance relays in the detection of low fault current, but it cannot be set instantaneous, it is set to trip very slow [14].

Moreover, most of the mentioned methods have ignored discussing the high impedance fault (HIF). In fact, HIFs mostly occur at distribution voltages and is a severe problem at lower voltages systems. However, higher voltages system such as a transmission line could be exposed to HIF, but it is less severity compared to the distribution system[15]. High impedance fault generally (HIF) occur when an energized conductor breaks and comes in contact with the ground through third-party object via a high impedance such as concrete, asphalt road, sand or tree branches [16]. Different schemes have been developed for detecting and handling the HIF, few of them who have cared with HIF identification under the presence of static voltage compensator (SVC) devices. Among these schemes are wavelet transform as in $[17,18]$, they proposed a new algorithm for High Impedance Fault (HIF) protection in high voltage transmission lines with the aid of wavelet packet transform (WPT). Wavelet transform has been employed with the artificial intelligence (AI) to detect HIF in [19-23]. However, neural network cannot learn during their online operation and wavelet require a deep knowledge of high-low filters and mother wavelet. Pattern recognition has combined with the wavelet transform and then used for detecting the HIF in [24], usually WT used for extracting the fault feature and pattern recognition used for classing the fault [25]. As well as, in [26] Stack-well transform (ST) is used for extracting the fault feature and then pattern recognition for assessing HIF. This scheme has high immunity to the unbalanced conditions but it is requiring high sampling rates. The scheme in [27] used the mathematical morphology (MM) and a data mining-based decision tree (DT) for HIF protection algorithms. A new scheme for HIF detection is proposed in [3], it relies on HIF-induced distortion of voltage and current waveforms to detect the fault. Scheme in [28] proposed a backup protection scheme in unified power flow compensator (UPFC) line, it is used the power differential feature from the apparent power flow in each phase at the local and remote bus to discriminate the fault condition.

As the result, the proposed method is suggested to detect the HIF in TCSC-compensated transmission line. The proposed method relies on the calculation standard deviation of the square error between the current signals during the fault period and current signals during the health state over the maximum values of the reference current signals to extract the fault feature. Consequently, during the fault condition the square error ratio is significantly exist, and stable in small values during the health state. The cumulative sum is then employed for enlarging the fault feature and a convenience fault detection index is being obtained. The main contribution of the proposed scheme represented in the integrating the static filter (standard deviation of the square error over the maximum value) with the cumulative scheme. The standard deviation of the square error is beneficial for sensing the small deviation in the 
current signals that results due to the HIF. The cumulative scheme is notable in term of speed of detecting abrupt in the signals due to the faults.

The paper is organized as follows. Section 1 introduces the work, section 2 describes the principle of the proposed method, section 3 presents the simulation and test results assessment section 4 concludes the work.

\section{THE PRINCIPLE OF THE PROPOSED METHOD}

The standard deviation of the square error of two variable over reference-variable can be defined as follows:

$$
e R=\frac{\operatorname{Std}(x-y)^{2}}{\max (\mathrm{y})}
$$

Where Std the standard deviation, $e R$ is the square error of two variable $x, y$, whereas $x$ is taken to be the current signals during the fault period, and $y$ is taken to be the reference value, it represents the current signals during the health state. The sampling rate is taken $4 \mathrm{kHz}$, and then the sum of $e R$ over the cycle can be calculated as follows

$$
E R_{k}=\left|\sum_{j=k-m+1}^{k} e R(j)\right|
$$

where $m$ represents the number of samples per the cycle; $j \& k$ are the instantaneous samples and sampling instant respectively. In general, during the normal operating condition $E R$ is stable around specific value, and have significant values during the fault interval. The cumulative approach is being used for amplifying the fault feature to generate the fault detection index, it can be expressed as follows:

$$
f d x=\max \left[f d x_{i-1}+E R_{i}-\lambda, 0\right]
$$

$f d x$ represents the fault detection index, $f d x_{(i-1)}$ represents the starting point where equal zero, $\lambda$ is a threshold. It is noticed that, during the health state output of equation (3) is zero since $E R$ not the threshold $\lambda$. However, during the fault condition $E R>\lambda$ and then the output is positive.

\section{SIMULATION AND TEST RESULTS ASSESSMENT}

The proposed method has been tested under different types of faults with different circumstances. Fig. 2 shows the power system model that established in PSCAD/EMTDC. It involves two power sources $500 \mathrm{kV}, 50 \mathrm{~Hz}$ connected through $200 \mathrm{~km}$ SVC-compensated overhead lines as shown in Fig. 2. TCSC unit has been installed at the middle of the transmission line, it is assumed to inject voltage in mid-point of the line. The current signals are tested at the sampling rate of $4 \mathrm{kHz}$, and then the data exported to MATLAB to perform the faults detection process.

High impedance faults (HIFs) are recruited as a single-line-to-ground faults occurs when a single conductor breaks and comes in contact with the ground through third-party object via a high impedance[3]. Such fault has low current magnitudes sometimes the current falls below the pickup threshold of overcurrent relays[29]. In this paper, we applied the HIF model that utilized in $[20,30]$, it is consist two amplified diode $D_{p}$ and $D_{n}$, two DC sources $V_{p}$ and $V_{n}$, and variable resistance $R_{p}$ and $R_{n}$ as seen in Fig. 3. The dc voltage is randomly varying around $0.01 \mathrm{~ms}$ whenever the system voltage is greater than $V_{p}$, current will flow towards the ground, reverse when the system voltage is less than Vn and no current will flow when the system voltage is greater than $\mathrm{Vn}$ and less than $\mathrm{V}_{\mathrm{p}}$.

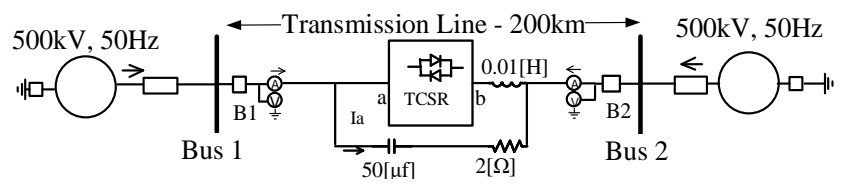

Fig. 1. Schematic diagram

\section{A. Proposed Method Assessment in uncompensated} power transmission line

To validate the performance of the proposed method under uncompensated power transmission line, consider a high impedance fault generated on phase-A at $110 \mathrm{~km}$ away from Bus 1 . The fault case started at $1.25 \mathrm{~s}$ and continued for $100 \mathrm{~ms}$ after the fault inception. Fig. 3 (a) illustrates the current waveforms, Fig. 3 (b) shows the square error ratio trajectories " $e R$ " and Fig. 3(c) shows the fault detection index " $f d x$ ". From Fig. 3(b) observed that, $e R$ trajectory at the pre-fault period stabled in specified limit and dramatically changed after the fault inception. 


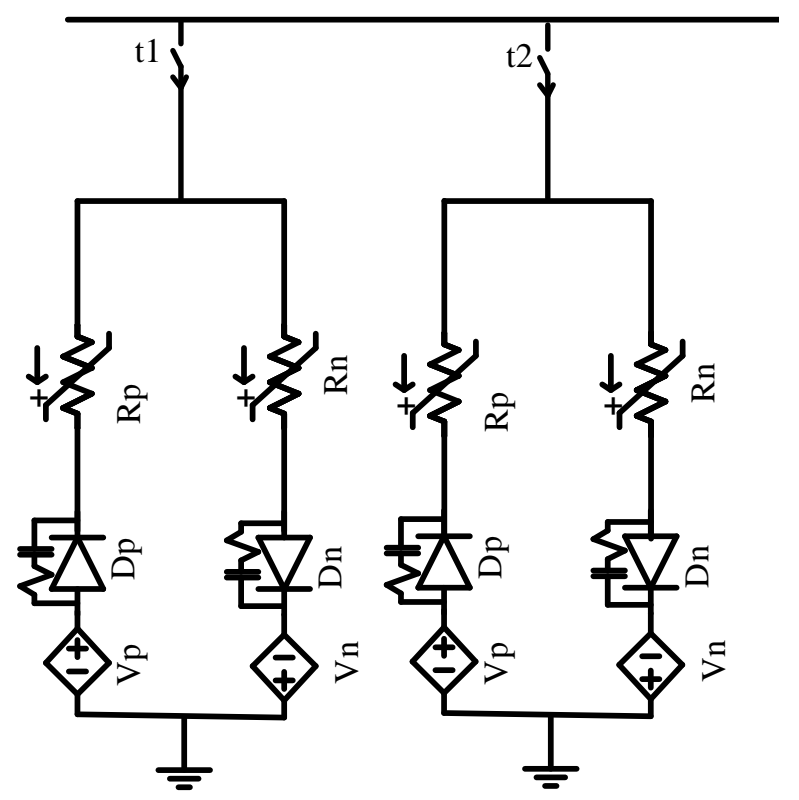

Fig. 2. High impedance model

From Fig. 3 (c) fault detection index " $f d x$ " took zero during the safe operating period and started taking high values after the fault inception. As we seen, the $f d x$ trajectory during the fault is much bigger compared to $e R$ trajectories as in Fig. 3(b), it is due to cumulative approach. The trajectory $f d x$ started taking high values at $\mathrm{t}=1.2515$, which means the fault can be detected within $1.5 \mathrm{~ms}$ after the fault commencement.

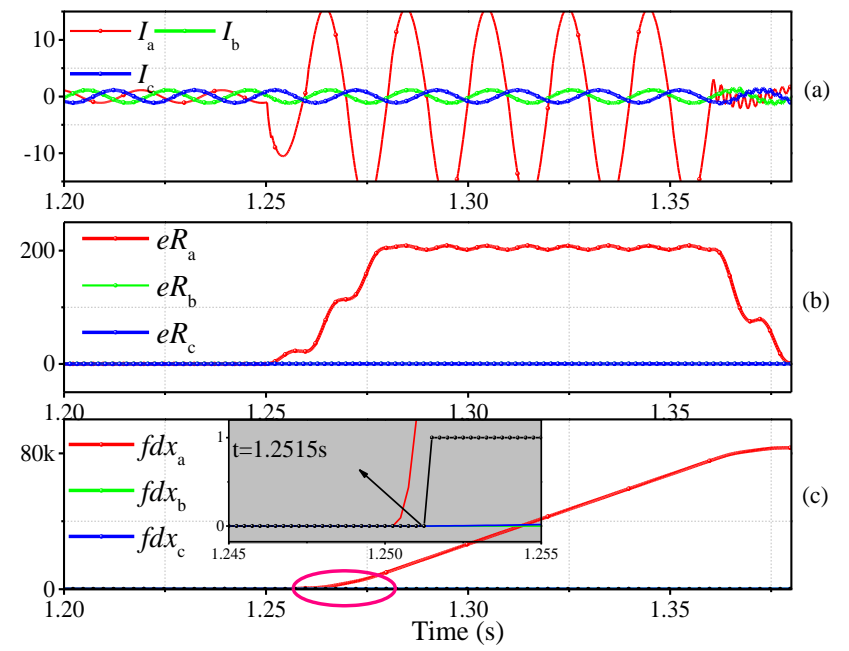

Fig. 3. Proposed method performance during Ag fault under uncompensated power line

B. Fault assessment during the existence of TCSC in the power transmission line

It is known that TCSC series-compensating device can provide transmission line with both the inductive reactance and capacitive reactance by controlling the firing pulses of thyristors. Therefore, the reach of the conventional distance relay may go-out of protection zone and causing over/under-reach operation due to presence of TCSC impedance in the fault loop. Fig. 4 shows the impedance trajectory when an $\mathrm{Ag}$ fault happened during different operation condition of TCSC. The fault case created at $110 \mathrm{~km}$ away from Bus 1 with fault resistance $10 \Omega$ started at $t=1.25 \mathrm{~s}$ and continued for $100 \mathrm{~ms}$ after the fault inception. It is obvious that, in the case of No compensation (the series capacitor is shorted) the impedance trajectory of Ag fault has been trapped inside the $80 \%$ zone as well as in case of $80 \%$ compensation. In the case of high compensation level (95\% compensation), the impedance trajectory of $\mathrm{Ag}$ fault has travelled out of protection zones $(80 \%$ zone) which is not supposed to happen. In this case, the TCSC module behaves like a parallel capacitor-inductor combination, and therefore, if the inductance was greater than the capacitance ( $\omega L>\frac{1}{\omega C}$ ); this case increases the equivalent-impedance. The impedance trajectory has passed away the $80 \%$ zone. So, the conventional distance protection may not work properly with presence of TCSC in the fault loop. Fortunately, such cases are easy to be identified by the proposed method as seen in Table I. Table I provides the corresponding $f d x$ values.

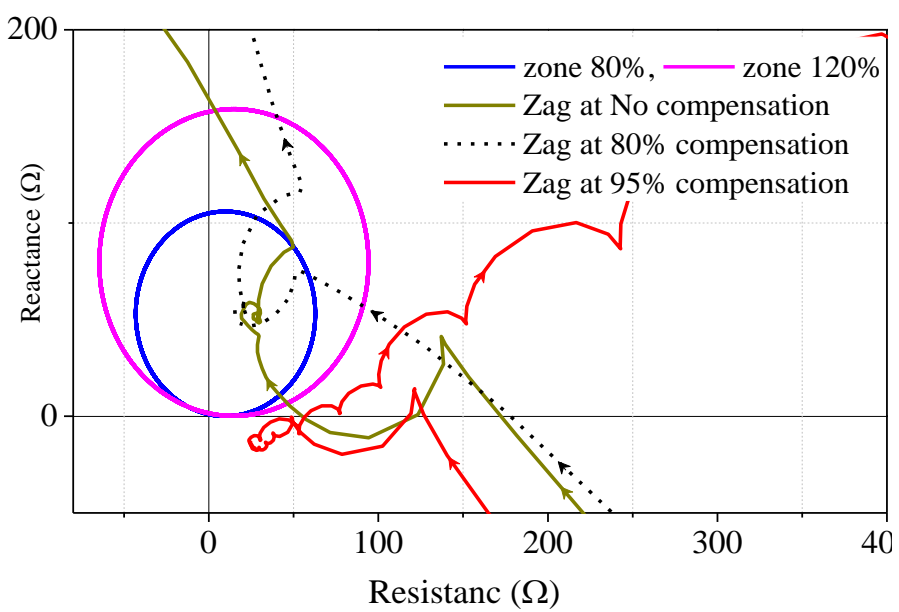

Fig.4. Impact of TCSC operation mode on the conventional Mho diagram

TABLE I: PROPOSED METHOD PERFORMANCE UNDER THE PRESENCE OF TCSC UNDER DIFFERENT TCSC OPERATING MODE

\begin{tabular}{llll}
\hline \hline TCSC operation mode & $f d x_{a}$ & $f d x_{b}$ & $f d x_{c}$ \\
\hline Without TCSC & 83302 & 0 & 0 \\
With compensation level 20\% & 83128 & 0 & 0 \\
With compensation level 80\% & 81402 & 0 & 0 \\
With compensation level 95\% & 78353 & 0 & 0 \\
\hline \hline
\end{tabular}


Although the fault circumstances are same just the compensation level of TCSC is different in test case. The $f d x$ values is vary according to the change in the firing angle of TCSC. This provide an extra difficulties to conventional scheme to detect fault under such condition. However, it is obvious that the fault cases were being detected satisfactory irrespective to the TCSC's compensation level.

\section{Fault happen close-in the terminals}

CT saturation can occurs when a close in fault to CT location at inception fault angles (FIA) $-90^{\circ}$ and $90^{\circ}$, because such fault can carry a high current with a DC offset. The proposed scheme has been tested under such condition, where CTs set with burden $5 \Omega$ and turn ratio 1000/5 were installed at sending end and receiving end. To test such fault consider a symmetrical fault occurs close in the sending end and receiving end respectively. The fault case started at 1.1s and continued for $100 \mathrm{~ms}$ with ground fault resistance $10 \Omega$. Fig. 5 (a, b, c) illustrates the current waveforms, $e R$, and $f d x$ trajectories respectively. Same fault scenarios is repeated at receiving terminals, Fig. $6(\mathrm{a}, \mathrm{b}, \mathrm{c})$ shows the proposed scheme performance. From the figures we can confirm that, the fault cases were detected correctly within $1 \mathrm{~ms}, 1.25 \mathrm{~ms}$ respectively.
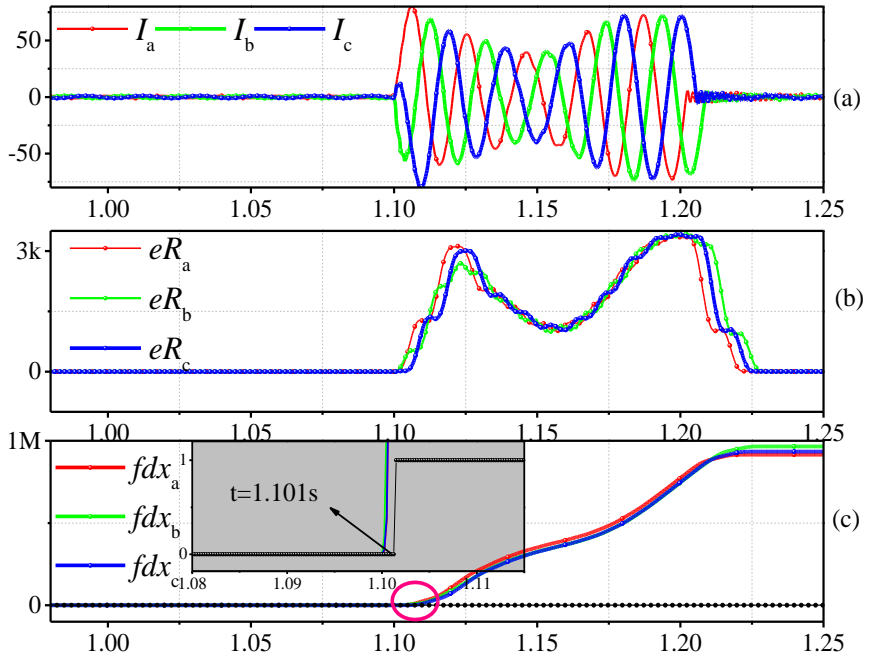

Fig. 5 Proposed scheme Performance during the close-in fault at sending terminals

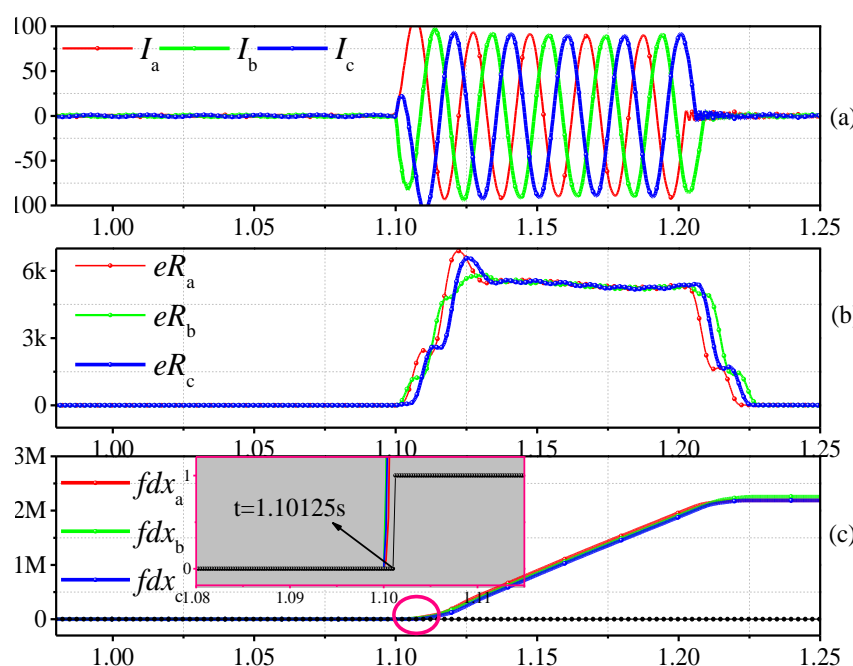

Fig. 6 Proposed scheme Performance during close-in fault at receiving terminals

\section{Noise Test}

Noise is the consequence of more or less random electrical signals getting coupled into circuits where they are unwanted. To perform this test, consider a high impedance fault subjected on phase-A and phase-C $150 \mathrm{~km}$ away from Bus 1 with compensation level $75 \%$. It is started at $\mathrm{t}=1.25 \mathrm{~s}$ and continued for $100 \mathrm{~ms}$ after the fault inception. Herein, phase A was corrupted with noise $20 \mathrm{~dB}$ while phase $\mathrm{B}$ and C are remained clear of noise. Fig. 7 (a, b, c) shows the current waveforms, $e R$, and $f d x$ trajectories respectively. It is obvious that from Fig. 7 (a) the noise has affected the current signals of phase- $B$, nevertheless the proposed scheme is succeeded to not consider the noise as a fault condition. Not only, the proposed scheme is satisfactorily detected the faults on phase-A and phase-B within $1.5 \mathrm{~ms}$ since the commencement of the fault. It is seen that the bad signalling of the current signals has not affected the final decision of the proposed method. Only $10.5 \mathrm{~ms}$ was enough to detect the fault case which would reinforce the exceptionality of the proposed method in such cases. 


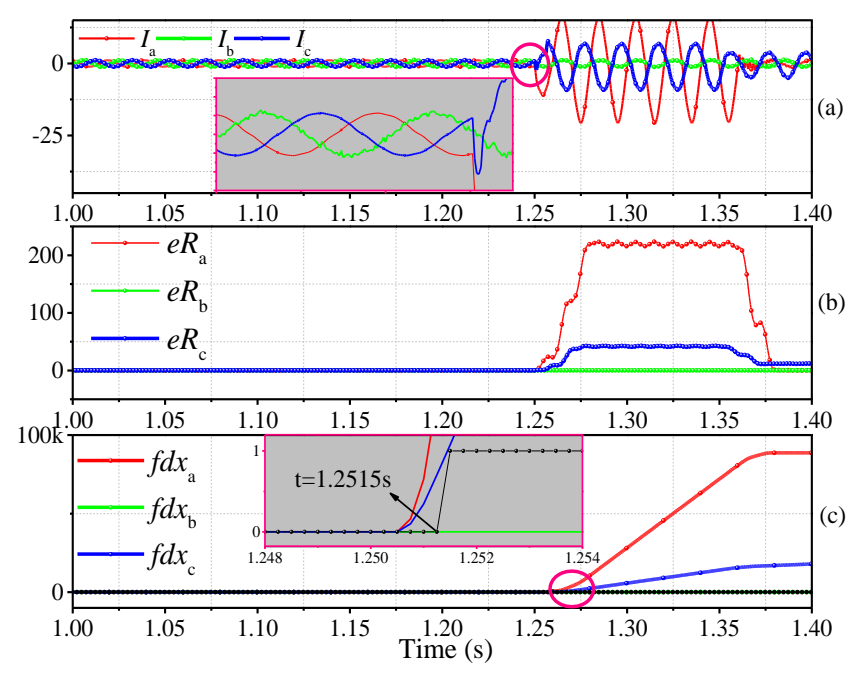

Fig. 7 Proposed scheme Performance during the noise condition

\section{E. Load flow change}

The Table II shows the proposed scheme performance during the power flow change. It is performed by changing the voltage sources angle ( $\delta 1 \approx$ sending end source angle, $\delta 2 \approx$ receiving end voltage source angle). High impedance fault subjected on phase-A located at $10 \mathrm{~km}$ back TCSC unit toward the sending end is selected to perform the test. The fault case started at $1.25 \mathrm{~s}$ and continued for $100 \mathrm{~ms}$ after the fault registered. It is evident that the changes in the voltage sources angle have no significant influence on the decision of the fault detection.

TABLE II: PERFORMANCE DURING POWER FLOW CHANGE

\begin{tabular}{lccll}
\hline \hline$\delta 1$ & $\delta 2$ & $f d x_{a}$ & $f d x_{b}$ & $f d x_{c}$ \\
\hline $30^{\circ}$ & $15^{\circ}$ & 87688 & 0 & 0 \\
$15^{\circ}$ & $25^{\circ}$ & 90930 & 0 & 0 \\
$30^{\circ}$ & $5^{\circ}$ & 89434 & 0 & 0 \\
\hline \hline
\end{tabular}

F. Proposed Method Assessment comparing to previous works

The aspects of high impedance fault occurs under the presence of TCSC in the fault loop is the main comparative factor with the cumulative approaches reported in [31-33]. These schemes are selected because the proposed method is considered a further development of cumulative schemes to be more reliable for faulty phase identification in TCSC-compensated transmission system. The fault case of high impedance fault that carried out at $110 \mathrm{~km}$ away from Bus 1 under high compensation is selected to perform the comparative assessment. Table III shows the comparison results between the proposed method and comparative schemes. It is seen that, the schemes [31-33] have failed to detect such fault, while the proposed scheme has detected the fault case accurately.

TABLE III: COMPARISON WITH PREVIOUS WORK

\begin{tabular}{lll}
\hline \hline Scheme & Assessment & Time response $(\mathrm{ms})$ \\
\hline$[31]$ & Failed & - \\
{$[32]$} & Failed & - \\
{$[33]$} & Failed & - \\
Proposed method & Success & $1.5 \mathrm{~ms}$ \\
\hline \hline
\end{tabular}

\section{CONCLUSION}

The proposed method is suggested to identify the high resistance faults in TCSC-compensated transmission system. It is found that the standard deviation of the square error between the current signals during the fault condition and current signals in the health state is significant during the fault interval. The cumulative sum is then applied to amplify this change in order to generate the fault detection index $(f d x)$. The proposed method has been tested several times through different fault circumstances. The proposed method characterized in the following aspect:

- Less measurement, and cover the entire line length

- Work properly with high impedance fault

- Good immunity against the noise in the current signals

- Remarkable time response.

The proposed scheme is unrestricted with the constraints of the conventional distance protection such as predefined the fault sections, fault type, in order to select an appropriate $\mathrm{K}$ factor. Additionally, the proposed scheme handled the faults correctly regardless the operating mode of TCSC compensation unit. Therefore, the proposed scheme is recommended to be integrated with distance protection and conventional overcurrent in power transmission line to enhance the reliability against the high impedance fault in the presence of TCSC-compensated line.

\section{REFERENCES}

[1] S. He, J. Suonan, and Z. Q. Bo, "Integrated Impedance-Based Pilot Protection Scheme for the TCSC-Compensated EHV/UHV Transmission Lines," IEEE Transactions on Power Delivery, vol. 28, pp. 835-844, 2013.

[2] R. K. Gajbhiye, B. Gopi, P. Kulkarni, and S. A. Soman, "Computationally Efficient Methodology for Analysis of Faulted Power Systems With Series-Compensated Transmission Lines: A Phase Coordinate Approach," IEEE Transactions on Power Delivery, vol. 23, pp. 873-880, 2008. 
[3] A. Soheili and J. Sadeh, "Evidential reasoning based approach to high impedance fault detection in power distribution systems," IET Generation, Transmission \& Distribution, vol. 11, pp. 1325-1336, 2017.

[4] O.H. Gupta and M. Tripathy, "Superimposed Energy-based Fault Detection and Classification Scheme for Series-compensated Line," Electric Power Components and Systems, vol. 44, pp. 1095-1110, 2016.

[5] P. Jafarian and M. Sanayepasand, "High-speed superimposed-based protection of series-compensated transmission lines," Iet Generation Transmission \& Distribution, vol. 5, pp. 1290-1300, 2011.

[6] S. Biswas, K. Kumar, A. Ghosal, and P. K. Nayak, "Fault detection and classification for TCSC compensated transmission lines using wavelet energy," in 2018 4th International Conference on Recent Advances in Information Technology (RAIT), 2018, pp. 1-5

[7] B. Vyas, R. P. Maheshwari, and B. Das, "Improved fault analysis technique for protection of Thyristor controlled series compensated transmission line," International Journal of Electrical Power \& Energy Systems, vol. 55, pp. 321-330, 2014.

[8] S. R. Samantaray, "A Data-Mining Model for Protection of FACTS-Based Transmission Line," IEEE Transactions on Power Delivery, vol. 28, pp. 612-618, 2013.

[9] S. R. Samantaray, "Decision tree-based fault zone identification and fault classification in flexible AC transmissions-based transmission line," IET Generation, Transmission \& Distribution, vol. 3, pp. 425-436, 2009.

[10] J. J. G. Ledesma, K. B. do Nascimento, L. R. de Araujo, and D. R. R. Penido, "A two-level ANN-based method using synchronized measurements to locate high-impedance fault in distribution systems," Electric Power Systems Research, vol. 188, p. 106576, 2020 .

[11] B. Sahoo and S. R. Samantaray, "An enhanced fault detection and location estimation method for TCSC compensated line connecting wind farm," International Journal of Electrical Power \& Energy Systems, vol. 96, pp. 432-441, 2018/03/01/ 2018.

[12] O. H. Gupta and M. Tripathy, "ERF-based fault detection scheme for STATCOM-compensated line," International Transactions on Electrical Energy Systems, vol. 27, p. e2314, 2017.

[13] C. Wang, G. Song, X. Kang, and J. Suonan, "Novel Transmission-Line Pilot Protection Based on Frequency-Domain Model Recognition," IEEE Transactions on Power Delivery, vol. 30, pp. 1243-1250, 2015.

[14] R. Matshidza, A. Bartylak, and R. Zivanovic, "Impact of high resistance faults on impedance protection perfomance," in CIRED 2005 - 18th International Conference and Exhibition on Electricity Distribution, 2005, pp. 1-5.

[15] M. S. Thomas, N. Bhaskar, and A. Prakash, "Voltage Based Detection Method for High Impedance Fault in a Distribution System," Journal of The Institution of Engineers (India): Series $B$, vol. 97, pp. 413-423, 2015.

[16] W. C. dos Santos, B. A. de Souza, N. S. D. Brito, F. B. Costa, and M. R. C. Paes, "High Impedance Faults: From Field Tests to Modeling," Journal of Control, Automation and Electrical Systems, vol. 24, pp. 885-896, 2013.

[17] A. Mahari and H. Seyedi, "High impedance fault protection in transmission lines using a WPT-based algorithm," International Journal of Electrical Power \& Energy Systems, vol. 67, pp. 537-545, 2015.

[18] S. AsghariGovar, P. Pourghasem, and H. Seyedi, "High impedance fault protection scheme for smart grids based on WPT and ELM considering evolving and cross-country faults," International Journal of Electrical Power \& Energy Systems, vol. 107, pp. 412-421, 2019.

[19] S. Silva, P. Costa, M. Gouvea, A. Lacerda, F. Alves, and D. Leite, "High impedance fault detection in power distribution systems using wavelet transform and evolving neural network," Electric Power Systems Research, vol. 154, pp. 474-483, 2018.

[20] I. Hafidz, P. E. Nofi, D. O. Anggriawan, A. Priyadi, and M. H. Pumomo, "Neuro wavelet algortihm for detecting high impedance faults in extra high voltage transmission systems," in 2017 2nd International Conference Sustainable and Renewable Energy Engineering (ICSREE), 2017, pp. 97-100.

[21] I. Baqui, I. Zamora, J. Mazón, and G. Buigues, "High impedance fault detection methodology using wavelet transform and artificial neural networks," Electric Power Systems Research, vol. 81, pp. 1325-1333, 2011.
[22] B. Vahidi, N. Ghaffarzadeh, S. H. Hosseinian, and S. M. Ahadi, "An approach to detection of high impedance fault using discrete wavelet transform and artificial neural networks," Simulation, vol. 86, pp. 203-215, 2010.

[23] S. Silva, P. Costa, M. Santana, and D. Leite, "Evolving neuro-fuzzy network for real-time high impedance fault detection and classification," Neural Computing and Applications, vol. 32, pp. 7597-7610, 2020.

[24] A.-R. Sedighi, M.-R. Haghifam, O. Malik, and M.-H Ghassemian, "High impedance fault detection based on wavelet transform and statistical pattern recognition," IEEE Transactions on Power Delivery, vol. 20, pp. 2414-2421, 2005.

[25] M. S. Ali, A. H. Abu Bakar, C. K. Tan, H. Arof, and H. Mokhlis, "High impedance fault detection and identification based on pattern recognition of phase displacement computation," IEEJ Transactions on Electrical and Electronic Engineering, vol. 13, pp. 549-560, 2018.

[26] M. Mishra, P. Routray, and P. k. Rout, "A Universal High Impedance Fault Detection Technique for Distribution System Using S-Transform and Pattern Recognition," Technology and Economics of Smart Grids and Sustainable Energy, vol. 1, 2016.

[27] K. Sekar and N. K. Mohanty, "Data mining-based high impedance fault detection using mathematical morphology," Computers \& Electrical Engineering, vol. 69, pp. 129-141, 2018.

[28] B. Kumar and A. Yadav, "Backup protection scheme for transmission line compensated with UPFC during high impedance faults and dynamic situations," IET Science, Measurement \& Technology, vol. 11, pp. 703-712, 2017.

[29] A. Ghaderi, H. L. Ginn III, and H. A. Mohammadpour, "High impedance fault detection: A review," Electric Power Systems Research, vol. 143, pp. 376-388, 2017.

[30] W. David Chan Tat and Y. Xia, "A novel technique for high impedance fault identification," IEEE Transactions on Power Delivery, vol. 13, pp. 738-744, 1998.

[31] M. R. Noori and S. M. Shahrtash, "Combined Fault Detector and Faulted Phase Selector for Transmission Lines Based on Adaptive Cumulative Sum Method," IEEE Transactions on Power Delivery, vol. 28, pp. 1779-1787, 2013.

[32] S. R. Mohanty, A. K. Pradhan, and A. Routray, "A Cumulative Sum-Based Fault Detector for Power System Relaying Application," IEEE Transactions on Power Delivery, vol. 23, pp. 79-86, 2008.

[33] M. H. Musa, Z. He, L. Fu, and Y. Deng, "Linear regression indexbased method for fault detection and classification in power transmission line," IEEJ Transactions on Electrical and Electronic Engineering, 2018.

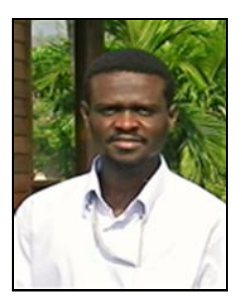

Dr. Mohammed Hussien Hassan Musa (1981), was born in Kadugli, Sudan, on May 29, 1981. He received a B.Sc. and M.Sc. degree in Electrical Engineering from Sudan University of Science and Technology in 2005 and 2010. He received a Ph.D. degree from Southwest Jiaotong University Nov 2018. He joined the National Electricity Corporation (NEC), Sudan 2006-2010. Presently, he work as assistant professor in college of engineering at Almughtaribeen University, and as senior technical support at Sudanese Thermal Power Generating Company (STPG) up to now.

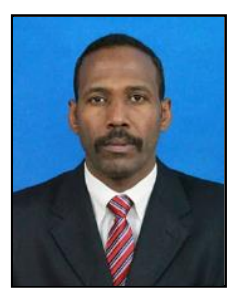

Dr. Abu Sabah I. A. Ahmed is assistant professor at the Department of Electrical Engineering, Karary University. He received the M.Sc. degree and Ph.D. degree in Electronic Engineering from University of Electronic Science and Technology of China in 2013 and 2017. His research area includes Robotics Control, Predictive and Adaptive Control and Neural Networks. Dr. Now he is teaching neural networks and Embedded Systems design for graduate students, and Control Theory, Automatic Control, Modern Control, Optimal Control and Electronic Measurements for junior students at Karary University. 